\title{
Dynamic Simulation of Heterogeneous Catalysis at Particle Scale to Estimate the Kinetic Parameters for the Pore Diffusion Model
}

\author{
Ameer Khan Patan ${ }^{1}$, Mallaiah Mekala'2, Sunil Kumar Thamida ${ }^{1 *}$ \\ ${ }^{1}$ Department of Chemical Engineering, National Institute of Technology, Warangal, \\ Telangana State 506004, India \\ ${ }^{2}$ Department of Chemical Engineering, B.V. Raju Institute of Technology, Narsapur, \\ Telangana, 502313, India
}

Received: $18^{\text {th }}$ January 2018; Revised: $26^{\text {th }}$ April 2018; Accepted: $8^{\text {th }}$ May 2018;

Available online: 14 $4^{\text {th }}$ November 2018; Published regularly: December 2018

\begin{abstract}
In this work, dynamic simulation at particle scale is carried out to predict the kinetics of solid catalyzed esterification reaction between acetic acid and methanol to produce methyl acetate and water. The reaction kinetic data utilized for modeling and validation is with solid catalyst as Indion 180. It was observed that the reaction rate and kinetics inside the pores of the catalyst is of higher magnitude as compared to bulk liquid. Each solid catalyst particle is surrounded by reactant solution of equal volume. A dynamic simulation is carried out using COMSOL Multiphysics which has solver for diffusion-reaction equation for both in liquid phase and inside porous catalyst particle. The intrinsic reaction rate constants for bulk liquid phase and inside the particle are obtained by solving the full diffusion-reaction equation and optimization method. Three different models (model 1,2,3) were proposed for evaluating the rate constants from the experimental kinetic data. The three models differ in the way the boundary condition of acetic acid concentration is defined at the interface of a catalyst particle and its immediate surrounding liquid. The different models originated based on the possibility of numerical solution to partial differential equations pertaining to particle scale catalytic reactions as distributed parameter models by various software such as MATLAB and COMSOL multiphysics. They also differ in the way the initial kinetics is utilized in evaluating the rate constants for outside and inside the catalyst particle. One of the proposed models (model 3) has shown good agreement with the experimental data. Copyright (C 2018 BCREC Group. All rights reserved
\end{abstract}

Keywords: Diffusion; Reaction; Esterification; Kinetics; Simulation; Catalysis

How to Cite: Patan, A.K., Mekala, M., Thamida, S.K. (2018). Dynamic Simulation of Heterogeneous Catalysis at Particle Scale to Estimate the Kinetic Parameters for the Pore Diffusion Model. Bulletin of Chemical Reaction Engineering \& Catalysis, 13 (3): 420-428 (doi:10.9767/bcrec.13.3.2098.420-428)

Permalink/DOI: https://doi.org/10.9767/bcrec.13.3.2098.420-428

\section{Introduction}

In solid catalyzed heterogeneous reaction there is a simultaneous reaction and diffusion occurring in the catalyst particles. Such a system is generally difficult to simulate or obtain

\footnotetext{
* Corresponding Author.

E-mail: sunil.chemengg@gmail.com (Thamida, S.K.)
}

analytical expression for overall kinetics of reaction conversion. Simulation of reaction diffusion equation was carried out and found to be useful to obtain internal mass transfer coefficients in porous catalysts [1]. Simultaneous mass transfer and reaction were also simulated for porous media such as catalytic monoliths [2]. Drug delivery model was carried out successfully using COMSOL Multiphysics [3]. Modelling of esterification of acrylic acid with ethanol in presence of 
both homogenous and heterogeneous catalysts using activity based model was also carried out [4]. An equilibrium stage wise model was used to validate esterification of $n$-butanol with acetic acid in presence of Amberlyst-15 catalyst [5]. Difficulty in obtaining rate constants for heterogeneous catalysis is also recently reported [6]. Catalyst efficiency in micro-reactors was evaluated by using computational fluid dynamics (CFD) simulation [7]. The effect of pore geometry on reaction kinetics in catalytic converter is also modeled and simulated using reaction diffusion and convection equation [8].

Esterification of acetic acid with methanol using porous solid catalyst particle is one such example as described previously [10]. It was one of the first papers to model esterification reaction using pore diffusion model. In this previous work there is an assumption of quasi steady state in simulating the dynamics of concentration profile inside the catalyst particle. The liquid side was assumed to be well stirred and hence a uniform concentration was attributed to all the species. This forms a dirichlet boundary condition instead of flux continuity which is addressed in the present work. The general reaction is:

$$
\mathrm{CH}_{3} \mathrm{COOH}(\mathrm{A})+\mathrm{CH}_{3} \mathrm{OH}(\mathrm{B}) \Leftrightarrow \mathrm{CH}_{3} \mathrm{COOCH}_{3}(\mathrm{C})+\mathrm{H}_{2} \mathrm{O}(\mathrm{D})
$$

A hydrodynamic flow pattern is developed in the dispersion of the catalyst particles and liquid reactants at sufficiently high RPM of the magnetic stirrer. At such high rates of mixing it is reasonable to assume a zero relative velocity between the catalyst particles and liquid reactants. And at such high RPM's the external mass transfer resistance is negligible and there is no much effect in conversion of acetic acid beyond certain rotations per minute (RPM) [11-13].

For this liquid state reaction there will be two rate constants one for the forward reaction and another for reverse reaction or it is equivalent to specify forward reaction rate constant $k_{f}$ and equilibrium constant $K_{e}$. The reaction rate expression assuming it as elementary reaction is:

$$
-r_{A}=k_{f}\left(C_{A} C_{B}-\frac{C_{C} C_{D}}{K_{e}}\right)
$$

This is applicable for homogeneous reactions but not for heterogeneous reactions due to diffusional limitation inside the catalyst particles. It is well established that $K_{e}$ equals 4.95 for Indion 180, an anionic resin catalyst [14]. Other research work also has determined the $K_{e}$ value as similar [15]. These catalyst particles are spherical in shape and with a diameter in the range of $400-1000 \mu \mathrm{m}$. The catalyst particles are porous in nature and hence the reactant liquids ingress into the catalyst particles, react and produce products which have to diffuse outward. Due to high catalytic activity inside the pores, the value of $k_{f}$ will be higher inside the catalyst particle than in the surrounding bulk liquid.

\section{Materials and Methods}

\subsection{Mathematical Modeling}

When certain number of catalyst particles are added to the reactant mixture as shown in Figure 1(a), during stirring the particles are uniformly distributed in the liquid and hence each of the particle is surrounded by certain amount of liquid as shown in Figure 1(b). Therefore to obtain the kinetics of heterogeneous catalytic reaction, the phenomena of reaction and diffusion occurring in this single cell consisting of one spherical catalyst particle surrounded by the liquid is sufficient for simulation purpose. For this purpose it requires the expression for $k_{f 1}$ in the liquid phase and $\mathrm{k}_{\mathrm{f} 2}$ in the solid phase. If there is a data of conversion $v s$ time $\left(X_{A}\right.$ vs $\left.t\right)$ for a reaction then it was shown for pore diffusion model that the initial rate of conversion $d X_{A} / d t$ at time 0 is a linear function of catalyst loading $w_{c}$ ( $\mathrm{g}$ of catalyst $/ \mathrm{cm}^{3}$ of liquid) as below [10].

$$
\frac{d X_{A}}{d t}=\beta_{\text {expt }} w_{c}+C_{A 0} k_{f l}
$$

Here $C_{A O}$ is the initial concentration of A. Hence from a plot of $d X_{A} / d t v s w_{c}$ will provide the value of $k_{f 1}$ from the intercept. Here $k_{f 1}$ is the rate constant on the liquid side. The slope

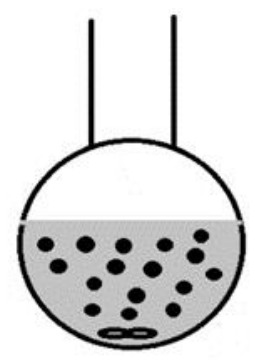

(a)

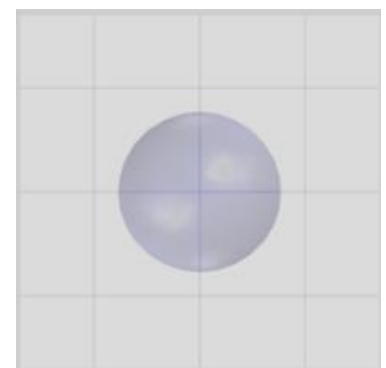

(b)
Figure 1. (a) Catalyst particles mixed in reactant product solution, (b) Catalyst particle surrounded by liquid 
$\beta_{\text {expt }}$ is related to the rate constant inside the particle $\mathrm{k}_{\mathrm{f} 2}$ by the following relationship $[9,10]$.

$\beta_{s i m}=\left(\frac{3 D_{A 2}}{R_{p}^{2} \rho_{p}}\right)\left(\frac{\partial C_{A}}{\partial r}\right)_{r=R p}$

Where $D_{A 2}{ }^{*}\left(\partial C_{A} / \partial r\right)$ represents the inward flux of A into the catalyst particle at its surface. The concentration profile $C_{A}(r)$ in Equation (5) is the solution to the following reaction-diffusion equation inside the catalyst particle.

$\frac{\partial C_{A}}{\partial t}=D_{A 2} \frac{1}{r^{2}} \frac{\partial}{\partial r}\left(r^{2} \frac{\partial C_{A}}{\partial r}\right)-\varepsilon\left[k_{f 2} C_{A} C_{B}-k_{b 2} C_{C} C_{D}\right]$

It depends on the choice of $k_{f 2}$ value which in turn provides such that $\beta_{\text {expt }}$ is matched. Given the possibilities of evaluating $k_{f 1}$ and $k_{f 2}$ at any temperature, the following three methods are delineated.

\subsection{Geometry}

The basic geometry created in the Comsol is a catalyst particle surrounded with certain volume of the reactant mixture. This kind of geometry is possible when the RPM of the stirrer inside the reactor is high such that the relative velocity between the catalyst particle and the reactant-product solution is almost equal to zero. However the density difference between catalyst particle and solution mixture is comparatively very small and hence there will be a uniform dispersion of catalyst particles. This actually signifies that convection term in the major governing equation, i.e. convectiondiffusion-reaction equation is negligible. The catalyst particles in reactant-product solution and the associated geometry in comsol is shown in Figure 2.

\subsection{Governing Equations}

The mathematical model that is used for this system is distributed parameter model involving time and space as independent variables and concentration or conversion as dependent variable. The generic form of reactiondiffusion equation for species $\mathrm{A}$ inside the catalyst particle whose pore volume fraction is equal to $\varepsilon$ is as follows:

$$
\frac{\partial C_{A}}{\partial t}-D_{A 2} \nabla^{2} C_{A}=-\varepsilon k_{f 2}\left[C_{A} C_{B}-\frac{C_{C} C_{D}}{K_{e}}\right]
$$

where $k_{f 2}$ and $k_{b 2}$ are the forward and backward
Table 1. Description of model for evaluation of rate constants

\begin{tabular}{|c|c|c|}
\hline & $K_{f 1}$ & $K_{f 2}$ \\
\hline Model 1 & $\begin{array}{l}\text { Homogeneous } \\
\text { kinetics without } \\
\text { catalyst }\end{array}$ & $\begin{array}{l}\text { Adjust or optimize } k_{f 2} \text { in } \\
\text { Equation (5) such that } \\
\beta_{\text {sim }} \text { from } \\
\text { Equation (4) averaged } \\
\text { over first } 15 \text { minutes } \\
\text { matches with } \beta_{\text {expt }} \text { from } \\
\text { Equation (3). And with } \\
\text { flux continuity at the } \\
\text { surface of the catalyst } \\
\text { particle. }\end{array}$ \\
\hline Model 2 & $\begin{array}{l}\text { From intercept of } \\
\text { Equation(1) }\end{array}$ & $\begin{array}{l}\text { Adjust or optimize } \mathrm{k}_{\mathrm{f} 2} \text { in } \\
\text { Equation (5) such that } \\
\beta_{\text {sim }} \text { from Equation (4) } \\
\text { evaluated at } t=0 \text { match- } \\
\text { es with } \beta_{\text {expt }} \text { from Equa- } \\
\text { tion (3). And with a } \\
\text { assumption that the } \\
\text { liquid side concentra- } \\
\text { tion of A is uniform } \\
\text { (dirichlet condition) is } \\
\text { used as in Mekala [10] }\end{array}$ \\
\hline Model 3 & $\begin{array}{l}\text { From intercept of } \\
\text { Equation (1) }\end{array}$ & $\begin{array}{l}\text { Adjust or optimize } k_{f 2} \text { in } \\
\text { Equation (5) such that } \\
\beta_{\text {sim }} \text { from Equation (4) } \\
\text { averaged over first } 15 \\
\text { minutes matches with } \\
\beta_{\text {expt }} \text { from Equation (3). } \\
\text { And with flux continui- } \\
\text { ty at the surface of the } \\
\text { catalyst particle. }\end{array}$ \\
\hline
\end{tabular}

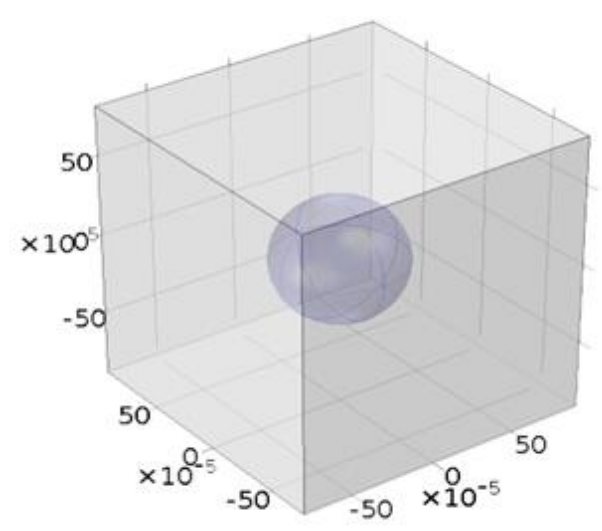

Figure 2. Geometry in COMSOL simulation for the catalyst particle surrounded by a cube of reactant liquid mixture. Units of length is in meters 
rate constants inside the catalyst particle and $\varepsilon$ is the available pore volume for the reaction to occur inside the catalyst particle. Since the pore volume is utilized for the reaction this type of model is called as pseudo homogenous model. Also since 1:1 mole ratio of $\mathrm{A}$ and $\mathrm{B}$ are taken as reactant mixture, it suffices to solve for species A only and rest of them are related through stoichiometry as in Equation (1). $C_{B}=$ $C_{A}, C_{C}=C_{A 0}-C_{A}$, and $C_{D}=C_{A 0}-C_{A}$. The conversion $X_{A}=1-C_{A} / C_{A 0}$. It is deduced from the assumption of equi-molar initial reactant mixture of $\mathrm{A}$ and $\mathrm{B}$ participating in the esterification reaction that at any time $C_{A}=C_{B}$. The change of densities of the liquids upon mixing and reaction is assumed negligible. Hence the volume of the reactant mixture is assumed constant. The catalyst Indion 180 is similar to Amberlyst. Since Indion 180 is available locally, it was procured for experimental study.

The generic form of reaction - diffusion equation in surrounding liquid phase is given in Equation (7).

$\frac{\partial C_{A}}{\partial t}-D_{A 1} \nabla^{2} C_{A}=-k_{f 1}\left[C_{A} C_{B}-\frac{C_{C} C_{D}}{K_{e}}\right]$

\subsection{Boundary Conditions and Initial Conditions}

At the center of the particle,

$$
r=0, \frac{\partial C_{A}}{\partial r}=0
$$

At surface of the particle,

$$
\begin{aligned}
& r=R_{p}, C_{A, \text { in }}=C_{A, \text { out }} \\
& r=R_{p},-D_{A 1} \frac{d C_{A}}{d r}=-D_{A 2} \frac{d C_{A}}{d r}
\end{aligned}
$$

At the faces of the cube, symmetry or no-flux condition is applied.

At $t=0, C_{A}=C_{A o}, 0<r<R_{p}$ and in the bulk liquid

\subsection{Simulation Tool}

Comsol Multiphysics is finite element based simulation software applicable for many kinds of engineering applications. It consists of different types of modules and selection of each module is application specific. The module we chose for our problem was transport of dilute species in 3D geometry as shown in Figure 2. Good solution of partial differential equations can be obtained using comsol and hence it is considered as powerful software package. It can be used in conjunction with MATLAB too.

\section{Results and Discussions}

\subsection{Homogeneous Rate Constant}

The conversion of acetic acid vs time for esterification without catalyst at various temperatures is plotted in the Figure 3 . The rate of homogeneous reaction is given by:

$$
\frac{\partial C_{A}}{\partial t}=-k_{f 1}\left[C_{A} C_{B}-\frac{C_{C} C_{D}}{K_{e}}\right]
$$

Since the experiments were conducted with equimolar ratio of $\mathrm{A} \& \mathrm{~B}$, the above equation reduces to Equation (13).

$$
\frac{d X_{A}}{d t}=k_{f 1} * C_{A 0}^{2}\left(\left(1-X_{A}\right)^{2}-\frac{X_{A}^{2}}{K_{e}}\right)
$$

By evaluating the rate of conversion $d X_{A} / d t$ at $t$ $=0$ which is the initial slopes of the curves in Figure 3, it provides the value of $k_{f 1}{ }^{*} C_{A 0}$, since $X_{A}=0$ at $t=0$. Further, $k_{f 1}$ is calculated at different temperatures. There is another way of obtaining $k_{f 1}$, that is from the solid catalyzed reaction kinetics as per Equation (3) from the $y$-intercept.

\subsection{Heterogeneous Rate Constant}

Simulation is done in order to optimize the value of $\mathrm{k}_{\mathrm{f} 2}$ as in Equation (6). Diffusivities of all species is assumed constant as $D_{A 1}=3 \times 10^{-9}$ $\mathrm{m}^{2} / \mathrm{sec}$ in the bulk liquid and $D_{A 2}=1 \times 10^{-9}$ $\mathrm{m}^{2} / \mathrm{sec}$ inside the catalyst particle since different diffusivities implies solving equations for all species. Reaction kinetics is used from

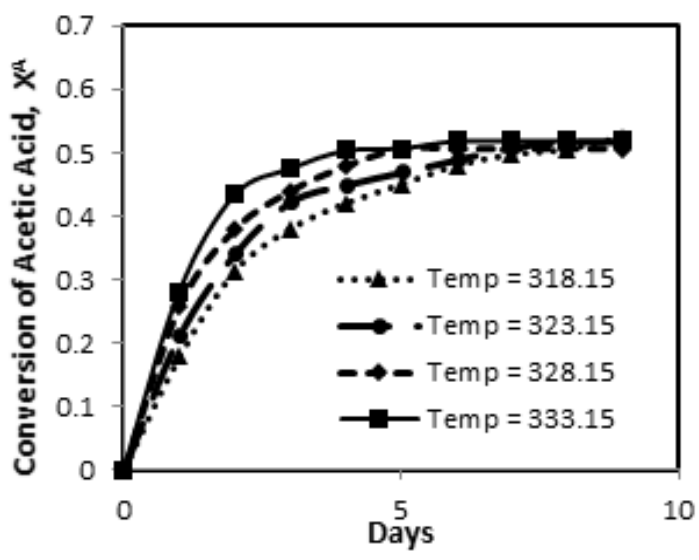

Figure 3. Conversion of acetic acid without catalyst at different temperatures 
[10]. The average concentration of $\mathrm{A}$ in the liquid phase is used to evaluate the overall conversion of $\mathrm{A}$. As described in Table 1, the plot between the error $=$ absolute $\left(\beta_{\text {sim }}-\beta_{\text {expt }}\right)$ and the guess value of $k_{f 2}$ is represented in Figure 4.

The values of $k_{f 1}$ and $k_{f 2}$ obtained from all the three models described in Table 1 are tabulated in Table 2 . The rate constants $k_{f 1}$ and $k_{f 2}$ are further fitted to Arrhenius rate laws as $k=$ $k_{0}{ }^{*} \exp \left(-E / R^{*} T\right)$. The linear fit for $k_{f 2}$ vs $1 / T \mathrm{ob}-$ tained from model 3 is plotted in Figure 5 . The values of pre-factor $k_{0}$ and activation energy $E$ are tabulated for $k_{f 1}$ and $k_{f 2}$ for each of the models in Table 3.

\subsection{Simulation Results}

Simulations were carried out with all the models described in earlier section and the results are discussed as follows:

Table 2. Rate constant values for all the three models at different temperatures

\begin{tabular}{cccc}
\hline $\begin{array}{c}\text { Tempera- } \\
\text { ture (K) }\end{array}$ & $\begin{array}{c}\text { Type of } \\
\text { Model }\end{array}$ & $\begin{array}{c}k_{f 1} \\
(\mathrm{~L} / \mathrm{mol} . \mathrm{min})\end{array}$ & $\begin{array}{c}k_{f 2} \\
(\mathrm{~L} / \mathrm{mol} . \mathrm{min})\end{array}$ \\
\hline 323.15 & Model 1 & 0.0000165 & 0.0603 \\
& Model 2 & 0.000414 & 0.1443 \\
& Model 3 & 0.000414 & 0.0603 \\
& & & \\
\hline 333.15 & Model 1 & 0.0000237 & 0.0895 \\
& Model 2 & 0.000673 & 0.1494 \\
& Model 3 & 0.000673 & 0.0895 \\
& & & \\
\hline 343.15 & Model 1 & 0.0000333 & 0.1298 \\
& Model 2 & 0.001064 & 0.1544 \\
& Model 3 & 0.001064 & 0.1298 \\
& & & \\
\hline 353.15 & Model 1 & 0.0000460 & 0.1843 \\
& Model 2 & 0.001638 & 0.1592 \\
& Model 3 & 0.001638 & 0.1843
\end{tabular}

3.3.1 Concentration distribution inside and outside catalyst particle

The spatial concentration distribution of acetic acid (component $\mathrm{A}$ ) at an instant of time during the dynamic simulation inside the geometry consisting of two domains, i.e. one in-

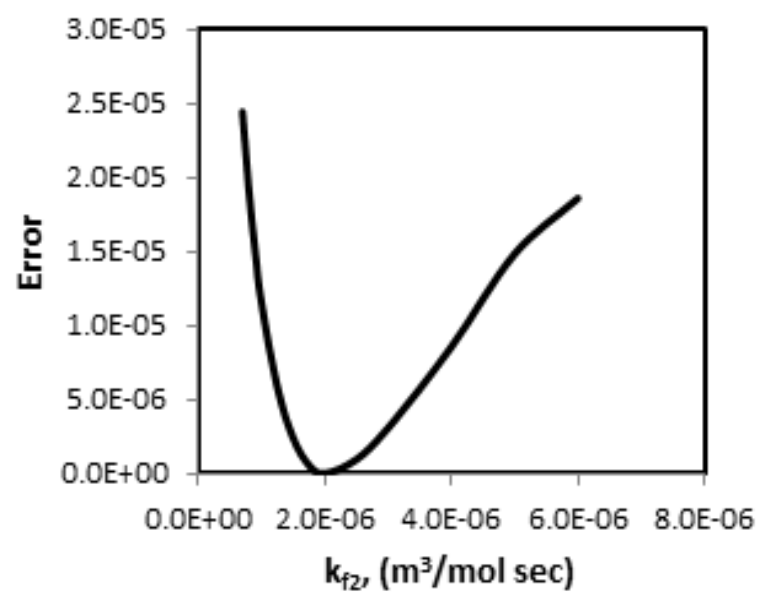

Figure 4. Error plot for evaluating optimum $k_{f 2}$ at a temperature of $343.15 \mathrm{~K}$ using Comsol solution for reaction-diffusion equation

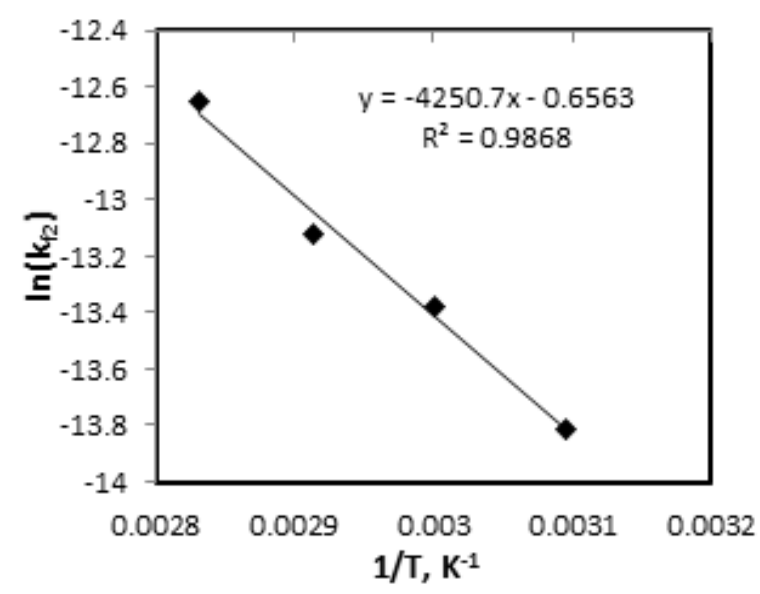

Figure 5. Arrhenius plot for reaction rate constant inside the catalyst pores

Table 3. The $k_{0}$ and $E$ values for $k_{f 1}$ and $k_{f 2}$ for all three models

\begin{tabular}{ccccc}
\hline \multirow{2}{*}{ Type of Model } & \multicolumn{2}{c}{ Rate Constant, $k_{f 1}$} & \multicolumn{2}{c}{ Rate Constant, $k_{f 2}$} \\
\cline { 2 - 5 } & $\begin{array}{c}\text { Pre Exponen- } \\
\text { tial factor, } k_{0} \\
(\mathrm{~L} / \mathrm{mol} \mathrm{min})\end{array}$ & $\begin{array}{c}\text { Activation } \\
\text { Energy, } E \\
(\mathrm{~J} / \mathrm{mol})\end{array}$ & $\begin{array}{c}\text { Pre Exponen- } \\
\text { tial factor, } k_{0} \\
(\mathrm{~L} / \mathrm{mol} \mathrm{min})\end{array}$ & $\begin{array}{c}\text { Activation Energy, } E \\
(\mathrm{~J} / \mathrm{mol})\end{array}$ \\
\hline Model 1 & 2.9659 & 32511.89 & 31126.02 & 35341.15 \\
Model 2 & 4465 & 43507 & 0.46 & 3115 \\
Model 3 & 4465 & 43507 & 31126.02 & 35341.15 \\
\hline
\end{tabular}


side the catalyst and the other bulk of the liquid is shown in Figure 6 with the help of slice in the Comsol software. As we know the conversion is high in the region inside the catalyst particle as compared to bulk of the liquid, the region inside the particle has lower concentration of the reactant species and in particular that of acetic acid (A). To bring out a clear picture of the concentration distribution in both the domains the concentration profile from one face of the cell to the other face passing through the catalyst at different times is shown in Figure 7.

\subsubsection{Validation of model}

The rate constants evaluated for all the three models as in Table 2 are used to determine the conversion of acetic acid at various temperatures and validated with that of experimental conversion of acetic acid $v$ s time. It was noticed that model 3 is in good agreement with that of experimental values. Model 3 is further validated to see the effect of conversion with respect to that of intrinsic parameters like different temperatures $(323.15-353.15 \mathrm{~K})$, different catalyst loading $\left(0.01-0.05 \mathrm{~g} / \mathrm{cm}^{3}\right)$ and different particle size $(400-1000 \mu \mathrm{m})$ and is listed in subsequent figures.

\subsubsection{Effect of temperature}

Temperature is considered to be as major intrinsic parameters for the evaluation of reaction kinetics. Increase in temperature brings out an instinctive thought that there will be increase in conversion caused either by increase in probability of collision between molecules or by fast diffusion of molecules towards active site of the catalyst where there are sufficient amount of $\mathrm{H}^{+}$ions to catalyze before giving rise to respective products. Another approach to compare mathematically is by Arrhenius expression which says that with increase of temperature the exponential value increases thereby the rate constant value increases. Increase in rate constant increases the rate of reaction as it is holds direct proportional relationship. All the three models were used to validate with respect to experimental investigations carried out at three different temperatures $333.15 \mathrm{~K}$, $343.15 \mathrm{~K}$, and $353.15 \mathrm{~K}$ for a particle diameter of $725 \mu \mathrm{m}$ and catalyst loading of $0.025 \mathrm{~g} / \mathrm{cm}^{3}$ and are shown in Figure 8, Figure 9, and Figure 10, respectively. It was observed that conversion reached equilibrium value very fast at high temperatures.

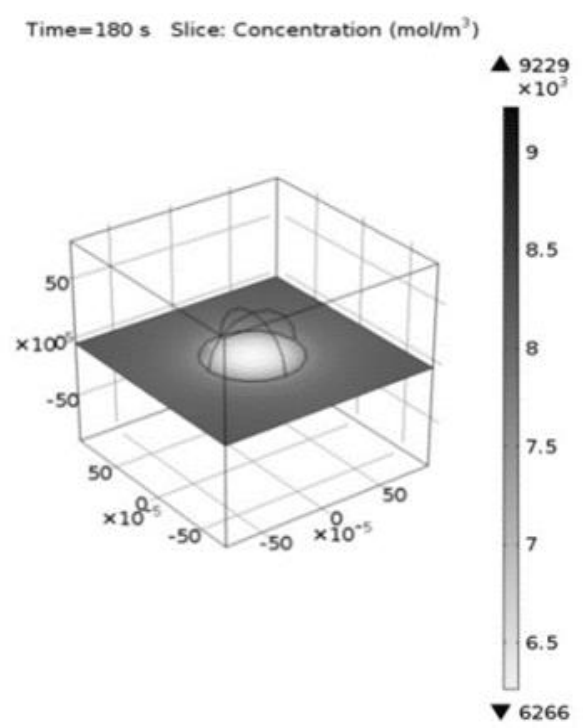

Figure 6. Spatial concentration distribution of acetic acid inside the catalyst particle and around it in the solution at an instant of time during dynamic simulation

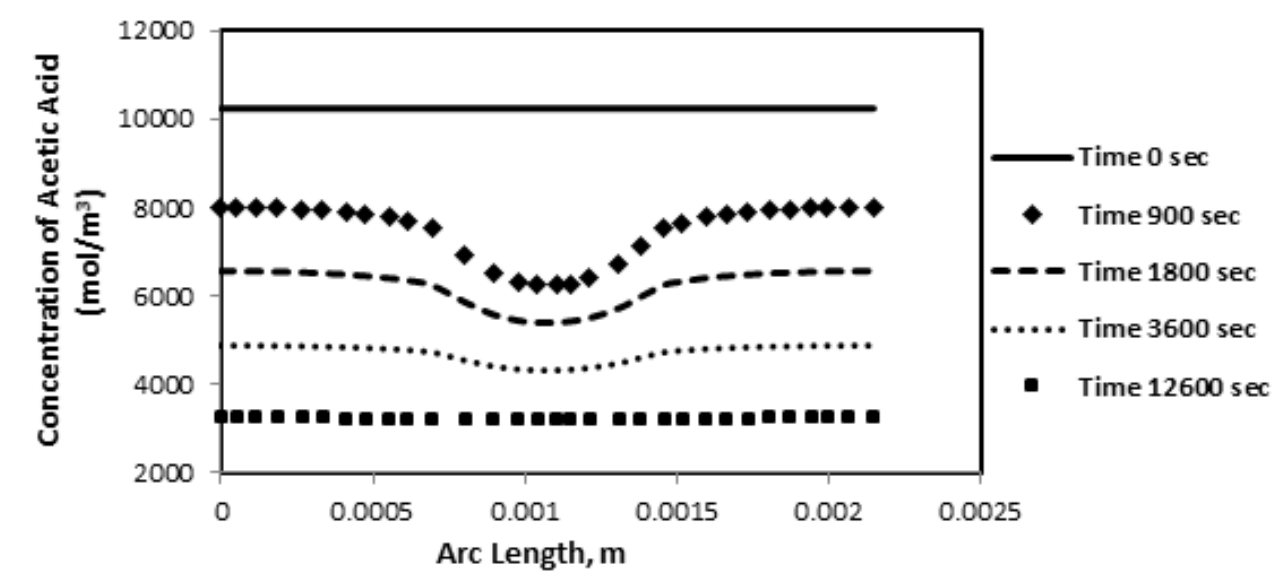

Figure 7. Concentration profiles along an arc length from one face of cell to opposite face passing through the catalyst at different times 
Further, model 3 which is in good agreement with that of experimental results is plotted separately to account the effect of conversion with respect to variation in temperature and is shown in Figure 11. These results enlighten that temperature controls the reaction to major extent and as it increases the time taken by the system to reach equilibrium is decreased.

\subsubsection{Effect of particle diameter}

The particle diameter effect keeping the same catalyst loading is also simulated. The re-

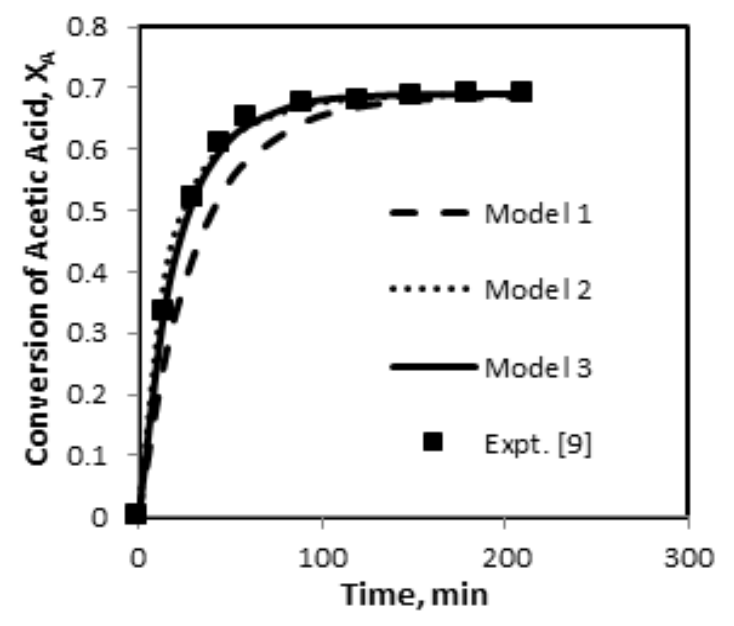

Figure 8. Kinetics of acetic acid conversion for temperature of $353.15 \mathrm{~K}$ at constant catalyst loading of $0.025 \mathrm{~g} / \mathrm{cm}^{3}$ and particle diameter of $725 \mu \mathrm{m}$ as calculated from experimental data [9] and predicted by the simulation

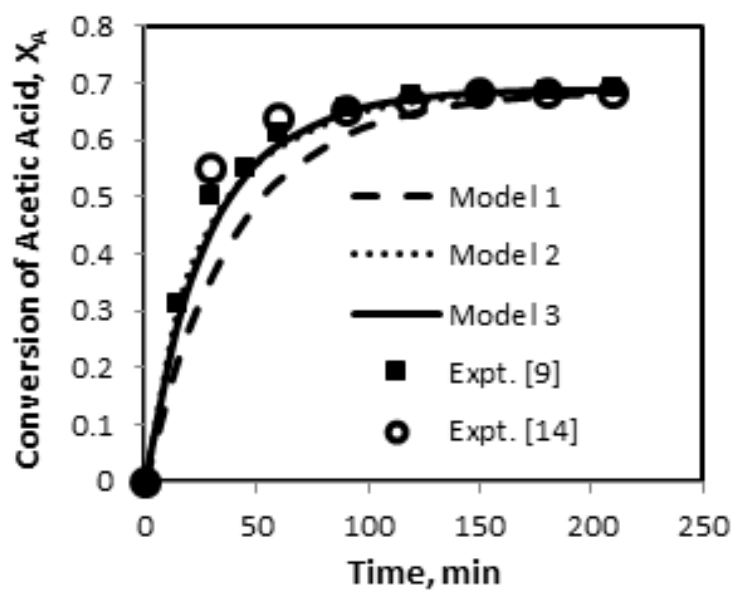

Figure 9. Kinetics of acetic acid conversion for temperature of $343.15 \mathrm{~K}$ at constant catalyst loading of $0.025 \mathrm{~g} / \mathrm{cm}^{3}$ and particle diameter of $725 \mu \mathrm{m}$ as calculated from experimental data $[9,14]$ and predicted by the simulation sults were obtained for three different particle sizes for a constant catalyst loading of 0.025 $\mathrm{gm} / \mathrm{cm}^{3}$ and at a temperature of $343.15 \mathrm{~K}$.

Interestingly there is no much variance in the kinetics owing particle size. This must be substituted with conventional explanation that reaction rate is proportional to $1 / R_{p}$, i.e. lower the particle size higher is the conversion and it must be attributed when the reaction takes place at the surface of the catalyst in a heterogeneous reaction. In the present case particle pore volume is also utilized and hence the entire volume of catalyst particles is same as long

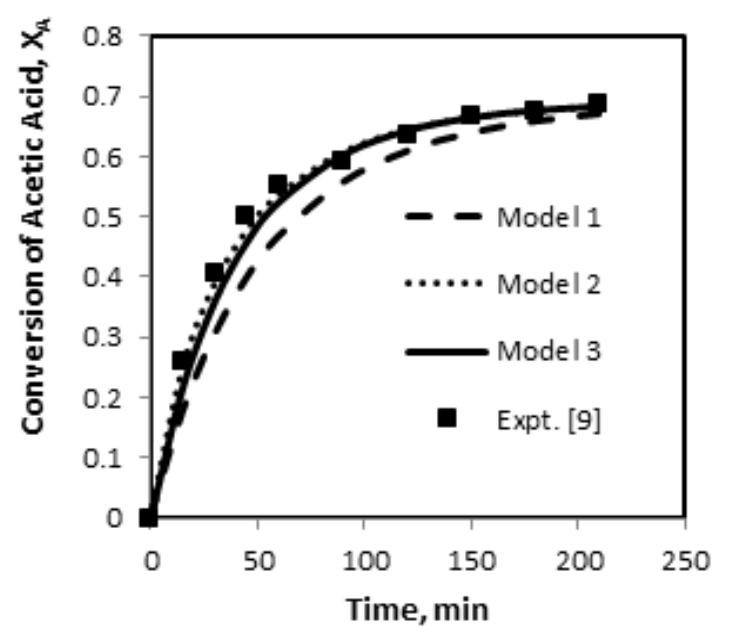

Figure 10. Kinetics of acetic acid conversion for temperature of $333.15 \mathrm{~K}$ at constant catalyst loading of $0.025 \mathrm{~g} / \mathrm{cm}^{3}$ and particle diameter of $725 \mu \mathrm{m}$ as calculated from experimental data [9] and predicted by the simulation

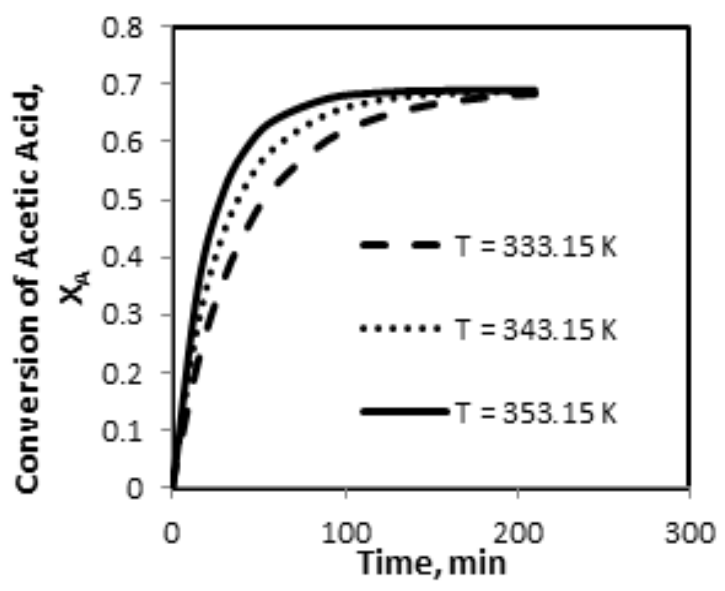

Figure 11. Kinetics of acetic acid conversion for different temperatures at constant catalyst loading of $0.025 \mathrm{~g} / \mathrm{cm}^{3}$ and particle diameter of $725 \mu \mathrm{m}$ as calculated from experimental data and predicted by the simulation using model 3 
as the catalyst loading is same. But the resultant dependency of the reaction rate over the catalyst particle size can be obtained by solving the reaction - diffusion equation inside the catalyst particle. Figure 12 shows the simulated results of kinetics of acetic acid conversion for different particle sizes and at a constant catalyst loading of $0.025 \mathrm{~g} / \mathrm{cm}^{3}$ and at a temperature of $343.15 \mathrm{~K}$.

\subsubsection{Effect of catalyst loading}

Simulations were also carried out at different catalyst loading for a temperature of $343.15 \mathrm{~K}$ and at a particle size of $725 \mu \mathrm{m}$. The kinetics effect with respect to catalyst loading is shown in Figure 13. When the amount of catalyst loading is increased for a particular size of particle the reaction rate increases because of more $\mathrm{H}^{+}$ions available for the catalysis and gives rise to constituent products. Increase in catalyst loading has a linear increase in conversion may be an erroneous conclusion. If catalyst loading is considered then particle size also needs to be addressed.

\section{Conclusions}

A hybrid model for heterogeneous reaction has been developed for carrying out the simulation of the esterification of acetic acid and methanol catalyzed by solids. The model incorporates mixed boundary condition of Dirichlet and flux continuity condition for species transport at the surface of the catalyst particle and Neumann boundary condition at the center

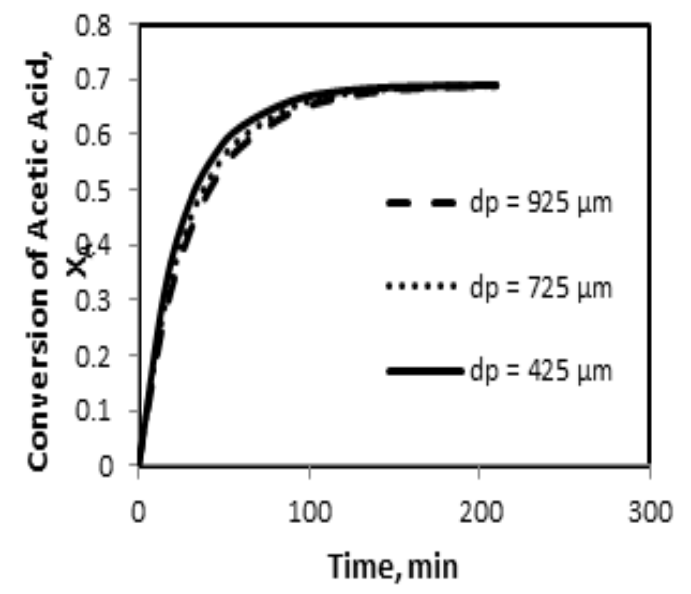

Figure 12. Kinetics of acetic acid conversion for various particle sizes at constant loading of $0.025 \mathrm{~g} / \mathrm{cc}$ as predicted by the simulation using $\mathrm{k}_{\mathrm{f} 1}$ evaluated with Matlab and $k_{f 2}$ evaluated with Comsol of a catalyst particle. This kind of boundary conditions lead to carrying out simulation more realistically. The concentration gradient at the surface of the catalyst helps in evaluating the rate constant inside the catalyst particle through simulation and by comparing with that of experimental data. The evaluated rate constant inside the catalyst particle and in bulk liquid is useful for understanding the conversion of acetic acid. It was observed that porous catalyst contribution to overall conversion of acetic acid is higher as compared to bulk of the liquid. The kinetic study was carried out using simulation for various parameters like particle size, catalyst loading, and temperature. It was found that increase in temperature increases the rate of reaction and increase in particle size for a constant catalyst loading decreases the reaction rate slightly. Further increase in catalyst loading for a particular particle size increases the conversion owing to higher catalyst volume.

\section{References}

[1] Joshi, S.Y., Harold, M.P., Balakotaiah, V. (2009). On the Use of Internal Mass Transfer Coefficients in Modeling of Diffusion and Reaction in Catalytic Monoliths. Chemical Engineering Science, 64: 4976-4991.

[2] Valdes-Parada, F.J., Aguilar-Madera, C.G., Alvarez-Ramirez, J. (2011). On Diffusion, Dispersion and Reaction in Porous Media. Chemical Engineering Science, 66: 2177-2190.

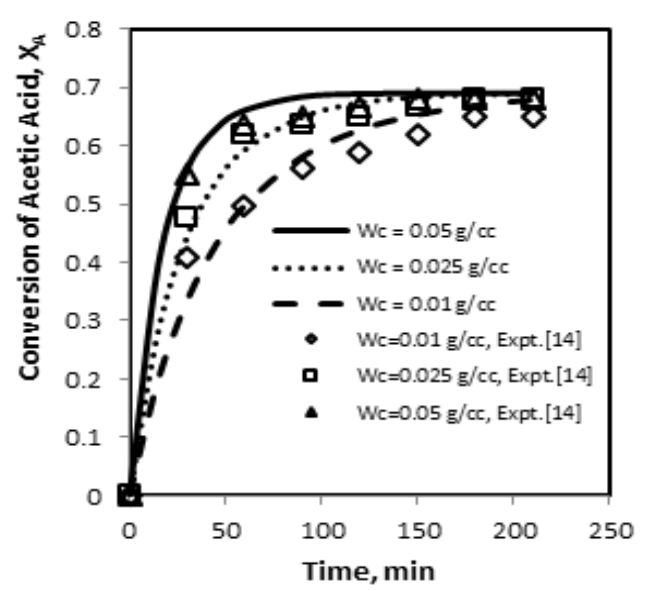

Figure 13. Kinetics of acetic acid conversion for various catalyst loading with particle diameter of $725 \mu \mathrm{m}$ as predicted by the simulation using $\mathrm{k}_{\mathrm{f} 1}$ evaluated with Matlab and $k_{f 2}$ evaluated with Comsol and compared with experimental data [14] 
[3] Moscicka-Studzinska, A., Ciach, T. (2012). Mathematical Modeling of Buccal Iontophoretic Drug Delivery System. Chemical Engineering Science, 80: 182-187.

[4] Jyoti, G., Keshav, A., Anandkumar, J., Bhoi, S. (2018). Homogeneous and Heterogeneous Catalyzed Esterification of Acrylic Acid with Ethanol: Reaction Kinetics and Modeling. International Journal of Chemical Kinetics, 50: 370-380.

[5] Steinigeweg, S., Gmehling, J. (2002). n-Butyl acetate synthesis via reactive distillation: thermodynamic aspects, reaction kinetics, pilot-plant experiments, and simulation studies. Industrial \& Engineering Chemistry Research, 41: 5483-5490.

[6] Pietrzyk, S.M., Redekop, E.A., Yablonsky, G.S., Marin, G.B. (2015). Precise Kinetic Measurements and Spatial Uniformity of Catalytic Beds. Chemical Engineering Science, 134: 367-373.

[7] Butcher, H., Wilhite, B.A. (2016). Enhancing Catalyst Effectiveness by Increasing Catalyst Film Thickness in Coated-Wall Microreactors: Exploiting Heat Effects in Catalytic Methane Steam Micro-reformers. Chemical Engineering Science, 143: 47-54.

[8] Mozaffari, B., Tischer, S., Votsmeier, M., Deutschmann, O. (2016). A One-Dimensional Modeling Approach for Dual Layer Monolithic Catalyst. Chemical Engineering Science, 139: 196-210.

[9] Mekala, M., Goli, V.R. (2014). Comparative kinetics of esterification of methanol-acetic acid in the presence of liquid and solid catalysts. Asia-Pacific Journal of Chemical Engineering, 9: 791-799.
[10] Mekala, M., Thamida, S.K., Goli, V.R. (2013). Pore Diffusion Model to Predict the Kinetics of Heterogeneous Catalytic Esterification of Acetic Acid and Methanol. Chemical Engineering Science, 104: 565-573.

[11] Titus, M.P., Bausach, M., Tejero, J., Iborra, M., Fite, C., Cunill, F., Izquierdo, J.F.(2007). Liquid-Phase Synthesis of Isopropyl TertButyl Ether by Addition of 2-Propanol to Isobutene on the over Sulfonate Dion-Exchange Resin Amberlyst-35. Applied Catalysis A:General, 323: 38-50.

[12] Ali, S.H., Tarakmah, A., Merchant, S.Q., AlSahhaf, T. (2007). Synthesis of Esters: Development of the Rate Expression for the Dowex 50 Wx8-400 Catalyzed Esterification of Propionic Acid with 1 Propanol. Chemical Engineering Science, 62: 3197-3217.

[13] Mao, W., Wang, X., Wang, H., Chang, H., Zhang, X., Han, J. (2008). Thermodynamic and Kinetic Study of Tert-amyl Methyl Ether (TAME) Synthesis. Chemical Engineering and Processing: Process Intensification, 47: 761-769.

[14] JagadeeshBabu, P.E., Sandesh, K., Saidutta, M.B. (2011). Kinetics of Esterification of Acetic Acid with Methanol in the Presence of Ion Exchange Resin Catalysts. Industrial and Engineering Chemistry Research, 50: 71557160 .

[15] Tsai, Y.T., Lin, H.M., Lee, M.J. (2011). Kinetic Behavior Esterification of Acetic Acid with Methanol over Amberlyst 36. Chemical Engineering Journal, 171: 1367-1372. 